\title{
INTEGRAL FORMULAE ON QUASI-EINSTEIN MANIFOLDS AND APPLICATIONS
}

\author{
A. BARROS and E. RIBEIRO JR*. \\ Departamento de Matemática-Universidade Federal do Ceará, 60455-760-Fortaleza-CE, Brazil \\ e-mail:abbarros@mat.ufc.br,ernani@mat.ufc.br
}

(Received 4 February 2011; revised 20 June 2011; accepted 22 September 2011)

\begin{abstract}
The aim of this paper is to extend for the $m$-quasi-Einstein metrics some integral formulae obtained in [1] (C. Aquino, A. Barros and E. Ribeiro Jr., Some applications of the Hodge-de Rham decomposition to Ricci solitons, Results Math. 60 (2011), 245-254) for Ricci solitons and derive similar results achieved there. Moreover, we shall extend the $m$-Bakry-Emery Ricci tensor for a vector field $X$ on a Riemannian manifold instead of a gradient field $\nabla f$, in order to obtain some results concerning these manifolds that generalize their correspondents to a gradient field.
\end{abstract}

2010 Mathematics Subject Classification. Primary 53C25, 53C20, 53C21; secondary 53C65.

1. Introduction. One of the motivation to study quasi-Einstein metrics on a Riemannian manifold $\left(M^{n}, g\right)$ is their close relation to Einstein metrics, which are warped products, see e.g. [4]. In this subject the $m$-Bakry-Emery Ricci tensor appears naturally. This tensor is given as follows:

$$
R i c_{f}^{m}=R i c+\nabla^{2} f-\frac{1}{m} d f \otimes d f
$$

where $0<m \leq \infty$, while Ric and $\nabla^{2} f$ stand for the Ricci tensor and the Hessian form, respectively. A natural generalisation for the previous tensor is to consider a vector field $X$ instead of a gradient of a smooth function $f$, more exactly, we define $\operatorname{Ric}_{X}^{m}$ as follows:

$$
\operatorname{Ric}_{X}^{m}=\operatorname{Ric}+\frac{1}{2} \mathcal{L}_{X} g-\frac{1}{m} X^{\mathrm{b}} \otimes X^{\mathrm{b}}
$$

where $X \in \mathfrak{X}(M), X^{\mathrm{b}}$ is the 1 -form associated to $X$, while $\mathcal{L}_{X} g$ stands for the Lie derivative of the vector field $X$.

A metric $g$ on a Riemannian manifold $\left(M^{n}, X\right)$ will be called $m$-quasi-Einstein metric, or simply a quasi-Einstein metric if the next relation

$$
R i c+\frac{1}{2} \mathcal{L}_{X} g-\frac{1}{m} X^{\mathrm{b}} \otimes X^{\natural}=\lambda g
$$

*Both partially supported by CNPq-BR. 
holds for some $\lambda \in \mathbb{R}$. In particular, we have

$$
\operatorname{Ric}(X, X)+\left\langle\nabla_{X} X, X\right\rangle=\frac{1}{m}|X|^{4}+\lambda|X|^{2} .
$$

Moreover, taking the trace of equation (1.3), we deduce

$$
R+\operatorname{div} X-\frac{1}{m}|X|^{2}=\lambda n \text {. }
$$

We point out that if $m=\infty$, then equation (1.3) reduces to the one associated to a Ricci soliton, as well as when $m$ is a positive integer and $X$ is a gradient vector field, it corresponds to warped product Einstein metrics, for more details see [5]. Following the terminology of Ricci soliton, a quasi-Einstein metric $g$ on a manifold $M^{n}$ will be called expanding, steady or shrinking, respectively, if $\lambda<0, \lambda=0$ or $\lambda>0$.

Definition 1. A quasi-Einstein metric will be called trivial if $X \equiv 0$.

The triviality definition is equivalent to saying that $M^{n}$ is an Einstein manifold. On the other hand, it is well known that on a compact manifold an $\infty$-quasi-Einstein metric with $\lambda \leq 0$ is trivial, see [6]. The same result was proved in [9] for quasi-Einstein metric on compact manifold with $m$ finite. Besides, we known that compact shrinking Ricci solitons have positive scalar curvature, see for example [6]. An extension of this result for shrinking quasi-Einstein metric with $X$ a gradient vector field and $1 \leq m<\infty$ was obtained in [5].

Before announcing the results we point out that they are generalisations of the results due to $[\mathbf{1}, \mathbf{1 0}]$ for Ricci solitons. Firstly, we have the following theorem.

THEOREM 1. Let $\left(M^{n}, g, X\right), n \geq 3$, be a compact Riemannian manifold satisfying $R i c_{X}^{m}=\lambda g$. Then $M^{n}$ is an Einstein manifold provided:

(1) $\int_{M} \operatorname{Ric}(X, X) \mathrm{d} \mathrm{M} \leq \frac{2}{m} \int_{M}|X|^{2} \operatorname{div} X \mathrm{dM}$.

(2) $X$ is a conformal vector field and $\int_{M} \operatorname{Ric}(X, X) \mathrm{dM} \leq 0$.

(3) $|X|$ is constant and $\int_{M} \operatorname{Ric}(X, X) \mathrm{d} M \leq 0$.

In order to proceed we remember a result due to Yau [11], which is a generalisation of Hopf's theorem: A subharmonic function $f: M^{n} \rightarrow \mathbb{R}$ defined over a complete noncompact Riemannian manifold is constant, provided its gradient belongs to $\mathrm{L}^{1}\left(M^{n}\right)$. Recently, this result was extended by Camargo et al. [3] for a vector field $X$. With the aid of this extension we derive the following result.

THEOREM 2. Let $\left(M^{n}, g, X\right)$ be a complete, non-compact Riemannian manifold satisfying Ric $_{X}^{m}=\lambda$. If $n \lambda \geq R$ and $|X| \in \mathrm{L}^{1}\left(M^{n}\right)$, then $M^{n}$ is an Einstein manifold.

Before proceeding, we make an observation: When $X=\nabla f$ is a gradient field, equation (1.5) enables us to write

$$
R+\Delta f=\frac{1}{m}|\nabla f|^{2}+\lambda n
$$

Thereby, we derive

$$
\langle\nabla f, \nabla R\rangle+\langle\nabla f, \nabla \Delta f\rangle=\frac{2}{m}\left\langle\nabla_{\nabla f} \nabla f, \nabla f\right\rangle
$$


2. Preliminaries. In this section we shall present some preliminaries which will be useful for the establishment of desired results. First we remember Lemma 2.1 due to [10].

LEMMA 1. Given a vector field $X$ on a Riemannian manifold $\left(M^{n}, g\right)$, we have

$$
\operatorname{div}\left(\mathcal{L}_{X} g\right)(X)=\frac{1}{2} \Delta|X|^{2}-|\nabla X|^{2}+\operatorname{Ric}(X, X)+D_{X} \operatorname{div} X .
$$

In particular, if $X=\nabla f$ is a gradient field, we have for all $Z \in \mathfrak{X}(M)$

$$
\operatorname{div}\left(\mathcal{L}_{X} g\right)(Z)=2 \operatorname{Ric}(Z, X)+2 D_{Z} \operatorname{div} X,
$$

or in $(1,1)$-tensorial notation

$$
\operatorname{div} \nabla \nabla f=\operatorname{Ric}(\nabla f)+\nabla \Delta f
$$

Remembering that the diffusion operator is given by $\Delta_{X}=\Delta-D_{X}$, the previous lemma allows us to deduce the following one.

Lemma 2. Let $\left(M^{n}, g, X\right)$ be a Riemannian manifold such that $\operatorname{Ric}_{X}^{m}=\lambda g$. Then we have

(1) $\frac{1}{2} \Delta|X|^{2}=|\nabla X|^{2}-\operatorname{Ric}(X, X)+\frac{2}{m}|X|^{2} \operatorname{div} X$.

(2) $\frac{1}{2} \Delta_{X}|X|^{2}=|\nabla X|^{2}-\lambda|X|^{2}+\frac{1}{m}|X|^{2}\left(2 \operatorname{div} X-|X|^{2}\right)$.

(3) If $M^{n}$ is compact and $\nabla X=0$, then $X=0$.

Proof. Since $\operatorname{div} g=0$, we deduce from the assumptions of the lemma that

$$
\operatorname{div} R i c+\frac{1}{2} \operatorname{div} \mathcal{L}_{X} g-\frac{1}{m} \operatorname{div}\left(X^{\triangleright} \otimes X^{b}\right)=0 .
$$

Next, we use the contracted second Bianchi identity, $\nabla R=2 \operatorname{div} R i c$, to arrive at

$$
\nabla R+\operatorname{div} \mathcal{L}_{X} g-\frac{2}{m} \operatorname{div} X X^{b}-\frac{2}{m}\left(\nabla|X|^{2}\right)^{b}=0 .
$$

In particular, for any $Z \in \mathfrak{X}(M)$ we have

$$
\langle\nabla R, Z\rangle+\operatorname{div}\left(\mathcal{L}_{X} g\right)(Z)-\frac{2}{m} X^{b}(Z) \operatorname{div} X-\frac{1}{m}\left(\nabla|X|^{2}\right)^{b}(Z)=0 .
$$

Therefore, for $Z=X$ we deduce

$$
\operatorname{div}\left(\mathcal{L}_{X} g\right)(X)=-\langle\nabla R, X\rangle+\frac{2}{m} \operatorname{div} X X^{\mathrm{b}}(X)+\frac{1}{m} \mathcal{L}_{X} g(X, X) .
$$

Next, we use the relation $\nabla R+\nabla \operatorname{div} X=\frac{1}{m} \nabla|X|^{2}$, jointly with equations (2.1) and (2.4) to arrive at

$$
\begin{aligned}
\frac{1}{2} \Delta|X|^{2}= & |\nabla X|^{2}-\operatorname{Ric}(X, X)-D_{X} \operatorname{div} X+\frac{1}{m} \mathcal{L}_{X} g(X, X)+D_{X} \operatorname{div} X \\
& -\frac{1}{m} X\left(|X|^{2}\right)+\frac{2}{m} \operatorname{div} X X^{b}(X) .
\end{aligned}
$$

Hence, we make use of Lemma 1 to conclude the first assertion of the lemma. 
Next, we notice that the second assertion is immediate from the first one just applying (1.4).

Supposing $\nabla X=0$, we have $|X|$ constant as well as $\operatorname{div} X=0$. Hence, the first item of the lemma yields $\operatorname{Ric}(X, X)=0$. Now we use equation (1.4) to deduce

$$
\frac{1}{m}|X|^{4}+\lambda|X|^{2}=0
$$

If $\lambda$ is non-negative we are done. Otherwise, let us assume $X \neq 0$ to arrive at a contradiction. In fact, equation (2.5) enables us to write $\lambda=-\frac{1}{m}|X|^{2}$. Thus, we obtain

$$
\operatorname{Ric}(X, Y)=\frac{1}{m} X^{\mathrm{b}}(X) X^{\mathrm{b}}(Y)-\frac{1}{m}|X|^{2} g(X, Y)=0,
$$

for any $Y$. So, we conclude that $M^{n}$ is Ricci flat. On the other hand, if we consider $Y$ a non-zero vector orthogonal to $X$, we get $\operatorname{Ric}(Y, Y)=\frac{1}{m}\left(\langle X, Y\rangle^{2}-|X|^{2}|Y|^{2}\right)=$ $-\frac{1}{m}|X|^{2}|Y|^{2}<0$, giving a contradiction. Then, $\lambda<0$, also implies $X=0$, which finishes the proof of the lemma.

Taking $X=\nabla f$ in the previous lemma and letting $\Delta_{f}=\Delta_{\nabla f}$, we derive the following corollary.

COROLlary 1. Under the assumptions of Lemma 2, if in addition $X=\nabla f$, then the following are true.

(1) $\frac{1}{2} \Delta|\nabla f|^{2}=|\nabla \nabla f|^{2}-\operatorname{Ric}(\nabla f, \nabla f)+\frac{2}{m}|\nabla f|^{2} \Delta f$.

(2) $\frac{1}{2} \Delta_{f}|\nabla f|^{2}=|\nabla \nabla f|^{2}-\lambda|\nabla f|^{2}+\frac{1}{m}|\nabla f|^{2}\left(2 \Delta f-|\nabla f|^{2}\right)$.

Writing equation (1.3) in the tensorial language

$$
R_{i j}+\nabla_{i} \nabla_{j} f-\frac{1}{m}(d f \otimes d f)_{i j}=\lambda g_{i j},
$$

we have the following lemma.

LeMma 3. Let $\left(M^{n}, g, \nabla f\right)$ be a Riemannian manifold such that $n \geq 3$ and $R i c_{\nabla f}^{m}=\lambda g$. Then the following formulae hold:

(1) $\frac{1}{2} \nabla_{i} R=\frac{m-1}{m} R_{i j} \nabla^{j} f+\frac{1}{m}(R-(n-1) \lambda) \nabla_{i} f$.

(2) $\nabla_{k} R_{i j}-\nabla_{j} R_{i k}=R_{i j k s} \nabla^{s} f+\frac{1}{m}\left(R_{i j} \nabla_{k} f-R_{i k} \nabla_{j} f\right)-\frac{\lambda}{m}\left(g_{i j} \nabla_{k} f-g_{i k} \nabla_{j} f\right)$.

(3) $\nabla\left(R+|\nabla f|^{2}-2 \lambda f\right)=\frac{2}{m}\left\{\nabla_{\nabla f} \nabla f+\left(|\nabla f|^{2}-\Delta f\right) \nabla f\right\}$.

Proof. For the first assertion we address the reader to formula (3.12) in Lemma 3.2 in [5]. Now we treat item (2). From equation (2.7) we infer

$$
\begin{aligned}
\nabla_{k} R_{i j}-\nabla_{j} R_{i k}= & -\left(\nabla_{k} \nabla_{j} \nabla_{i} f-\nabla_{j} \nabla_{k} \nabla_{i} f\right) \\
& +\frac{1}{m}\left(\nabla_{k} \nabla_{i} f \nabla_{j} f+\nabla_{k} \nabla_{j} f \nabla_{i} f-\nabla_{j} \nabla_{i} f \nabla_{k} f-\nabla_{j} \nabla_{k} f \nabla_{i} f\right) \\
= & R_{i j k s} \nabla^{s} f+\frac{1}{m}\left(R_{i j} \nabla_{k} f-R_{i k} \nabla_{j} f\right)-\frac{\lambda}{m}\left(g_{i j} \nabla_{k} f-g_{i k} \nabla_{j} f\right),
\end{aligned}
$$

where we interchanged the covariant derivatives to get item (2). 
Finally, we prove the last item of the lemma. In fact, from item (1) and equation (2.7) we deduce

$$
\begin{aligned}
\frac{1}{2} \nabla\left(R+|\nabla f|^{2}\right) & =\frac{m-1}{m} \operatorname{Ric}(\nabla f)+\frac{1}{m}(R-(n-1) \lambda) \nabla f+\nabla_{\nabla f} \nabla f \\
& =\operatorname{Ric}(\nabla f)+\nabla_{\nabla f} \nabla f-\frac{1}{m} \operatorname{Ric}(\nabla f)+\frac{1}{m}(R-(n-1) \lambda) \nabla f \\
& =\frac{1}{m}|\nabla f|^{2} \nabla f+\lambda \nabla f-\frac{1}{m} \operatorname{Ric}(\nabla f)+\frac{1}{m}(R-(n-1) \lambda) \nabla f .
\end{aligned}
$$

Thus, using $R-n \lambda=\frac{1}{m}|\nabla f|^{2}-\Delta f$ we achieve

$$
\begin{aligned}
\nabla\left(R+|\nabla f|^{2}-2 \lambda f\right) & =\frac{2}{m}\left\{\left(|\nabla f|^{2}+R-n \lambda+\lambda\right) \nabla f-\operatorname{Ric}(\nabla f)\right\} \\
& =\frac{2}{m}\left\{\left(|\nabla f|^{2}+\frac{1}{m}|\nabla f|^{2}-\Delta f+\lambda\right) \nabla f-\operatorname{Ric}(\nabla f)\right\} \\
& =\frac{2}{m}\left\{\left(|\nabla f|^{2}-\Delta f\right) \nabla f+\frac{1}{m}|\nabla f|^{2} \nabla f+\lambda \nabla f-\operatorname{Ric}(\nabla f)\right\} \\
& =\frac{2}{m}\left\{\left(|\nabla f|^{2}-\Delta f\right) \nabla f+\nabla_{\nabla f} \nabla f\right\},
\end{aligned}
$$

which concludes the proof of the lemma.

It is convenient to notice that for $m=\infty$ we derive the classical Hamilton equation [7] for a gradient Ricci soliton:

$$
R+|\nabla f|^{2}-2 \lambda f=C
$$

where $C$ is constant.

As a consequence of the preceding lemma we obtain the following corollary.

Corollary 2. Let $\left(M^{n}, g, \nabla f\right)$ be a Riemannian manifold such that $n \geq 3$ and $R i c_{\nabla f}^{m}=\lambda g$. Then the following formulae hold:

(1) $\frac{1}{2}\langle\nabla R, \nabla f\rangle=\frac{m-1}{m} \operatorname{Ric}(\nabla f, \nabla f)+\frac{1}{m}(R-(n-1) \lambda)|\nabla f|^{2}$.

(2) $\frac{1}{2}|\nabla R|^{2}=\frac{m-1}{m} \operatorname{Ric}(\nabla f, \nabla R)+\frac{1}{m}(R-(n-1) \lambda)\langle\nabla f, \nabla R\rangle$.

Proof. We choose $Z \in \mathfrak{X}(M)$ on item (1) of the quoted lemma to deduce

$$
\frac{1}{2}\langle\nabla R, Z\rangle=\frac{m-1}{m} \operatorname{Ric}(\nabla f, Z)+\frac{1}{m}(R-(n-1) \lambda)\langle\nabla f, Z\rangle .
$$

Therefore, the corollary follows.

Proceeding, we arrive at the main lemma of this section.

LemmA 4. Let $\left(M^{n}, g, \nabla f\right)$ be a Riemannian manifold satisfying $\operatorname{Ric}_{\nabla f}^{m}=\lambda g$. Then,

$$
\begin{aligned}
\frac{1}{2} \Delta R= & -\operatorname{Ric}(\nabla f, \nabla f)-\left|\nabla^{2} f-\frac{(\Delta f)}{n} g\right|^{2}-\frac{(\Delta f)^{2}}{n}+\lambda \Delta f+\langle\nabla R, \nabla f\rangle \\
& +\frac{1}{m}\left\{|\nabla f|^{2} \Delta f+\operatorname{div}\left(\nabla_{\nabla f} \nabla f-\nabla f \Delta f\right)\right\} .
\end{aligned}
$$


Proof. Initially we compute the divergence of identity (3) of Lemma 3 to obtain $\Delta R+\Delta|\nabla f|^{2}-2 \lambda \Delta f=\frac{2}{m}\left\{\left\langle\nabla\left(|\nabla f|^{2}-\Delta f\right), \nabla f\right\rangle+\left(|\nabla f|^{2}-\Delta f\right) \Delta f+\operatorname{div}\left(\nabla_{\nabla f} \nabla f\right)\right\}$.

Using Bochner's formula: $\frac{1}{2} \Delta|\nabla f|^{2}=\operatorname{Ric}(\nabla f, \nabla f)+\left|\nabla^{2} f\right|^{2}+\langle\nabla f, \nabla \Delta f\rangle$, and writing $\left|\nabla^{2} f\right|^{2}=\left|\nabla^{2} f-\frac{(\Delta f)}{n} g\right|^{2}-\frac{1}{n}(\Delta f)^{2}$, we have

$$
\begin{aligned}
\frac{1}{2} \Delta R= & -\operatorname{Ric}(\nabla f, \nabla f)-\left|\nabla^{2} f-\frac{\Delta f}{n} g\right|^{2}-\frac{(\Delta f)^{2}}{n}+\lambda \Delta f-\langle\nabla \Delta f, \nabla f\rangle \\
& +\frac{2}{m}\left\langle\nabla_{\nabla f} \nabla f, \nabla f\right\rangle+\frac{1}{m}\left\{\left(|\nabla f|^{2}-\Delta f\right) \Delta f-\langle\nabla \Delta f, \nabla f\rangle+\operatorname{div}\left(\nabla_{\nabla f} \nabla f\right)\right\} .
\end{aligned}
$$

Next, we invoke equation (1.6) to write

$$
\langle\nabla \Delta f, \nabla f\rangle=\left\langle\nabla\left(n \lambda+\frac{1}{m}|\nabla f|^{2}-R\right), \nabla f\right\rangle=\frac{2}{m}\left\langle\nabla_{\nabla f} \nabla f, \nabla f\right\rangle-\langle\nabla R, \nabla f\rangle .
$$

Then, the last relation for $\frac{1}{2} \Delta R$ turns into

$$
\begin{aligned}
\frac{1}{2} \Delta R= & -\operatorname{Ric}(\nabla f, \nabla f)-\left|\nabla^{2} f-\frac{\Delta f}{n} g\right|^{2}-\frac{(\Delta f)^{2}}{n}+\lambda \Delta f+\langle\nabla R, \nabla f\rangle \\
& +\frac{1}{m}\left\{\left(|\nabla f|^{2}-\Delta f\right) \Delta f-\langle\nabla \Delta f, \nabla f\rangle+\operatorname{div}\left(\nabla_{\nabla f} \nabla f\right)\right\} .
\end{aligned}
$$

At this point we use $\operatorname{div}(\nabla f \Delta f)=(\Delta f)^{2}+\langle\nabla \Delta f, \nabla f\rangle$ to achieve the formula in the statement, which finishes the proof of lemma.

\section{Proofs of the results stated in the introduction.}

3.1. Proof of Theorem 1. First we integrate identity (1) of Lemma 2 to infer

$$
\frac{1}{2} \int_{M} \Delta|X|^{2} \mathrm{dM}=\int_{M}|\nabla X|^{2} \mathrm{dM}-\int_{M} \operatorname{Ric}(X, X) \mathrm{dM}+\frac{2}{m} \int_{M}|X|^{2} \operatorname{div} X \mathrm{dM} .
$$

This yields

$$
\int_{M}|\nabla X|^{2} \mathrm{dM}=\int_{M} \operatorname{Ric}(X, X) \mathrm{dM}-\frac{2}{m} \int_{M}|X|^{2} \operatorname{div} X \mathrm{dM} .
$$

Since we are assuming that the right-hand side of (3.1) is less than or equal to zero, we obtain $\nabla X=0$. So, assertion (3) of Lemma 2 allows us to conclude the first item.

Proceeding, we know that there exists a smooth function $\rho$ on $M$, for which

$$
\mathcal{L}_{X} g=2 \rho g .
$$

In particular, $\left\langle\nabla_{X} X, X\right\rangle=\rho|X|^{2}$. Moreover, taking the trace of both members of equation (3.2) we also obtain

$$
\operatorname{div} X=n \rho .
$$


On the other hand, we notice that

$$
\begin{aligned}
\operatorname{div}\left(X|X|^{2}\right) & =|X|^{2} \operatorname{div} X+2\left\langle\nabla_{X} X, X\right\rangle \\
& =(n+2) \rho|X|^{2}
\end{aligned}
$$

Since $M^{n}$ is compact, we use Stokes' formula to obtain

$$
\int_{M} \rho|X|^{2} \mathrm{dM}=0
$$

Thereby, using this result jointly with relation (3.1), we conclude that $\nabla X=0$, since we are assuming $\int_{M} \operatorname{Ric}(X, X) \mathrm{d} \mathbf{M} \leq 0$. Therefore, using assertion (3) of Lemma 2, we conclude that $M^{n}$ is an Einstein manifold.

Finally, if $|X|$ is constant, we can apply Stokes' formula on equation (3.1) to derive

$$
\int_{M}|\nabla X|^{2} \mathrm{~d} \mathrm{M}=\int_{M} \operatorname{Ric}(X, X) \mathrm{d} \mathrm{M}
$$

From here we conclude the proof of the theorem.

REMARK 1. We notice that for $n=2$, we may write equation (3.1) as follows

$$
\int_{M}|\nabla X|^{2} \mathrm{dM}=\frac{1}{2} \int_{M} K|X|^{2} \mathrm{dM}-\frac{2}{m} \int_{M}|X|^{2} \operatorname{div} X \mathrm{dM},
$$

where $K$ stands for the Gaussian curvature. In particular we have:

- If $|X|$ is a non-null constant, then $M^{2}$ has genus zero or one.

- If $X$ is a non-trivial conformal vector field and $K$ is constant, then $M^{2}$ is isometric to $\mathbb{S}^{2}(r)$.

3.2. Proof of Theorem 2. Taking into account that $R i c_{X}^{m}=\lambda g$, then by equation (1.5) we arrive at

$$
m \operatorname{div} X=|X|^{2}+m(n \lambda-R)
$$

Thus, if $(n \lambda-R) \geq 0$, then we have $m \operatorname{div} X \geq 0$. On the other hand, if $|X| \in L^{1}(M)$, we may invoke Proposition 1 in [3] to derive that $\operatorname{div} X=0$. Next, we may use equation (3.7) to conclude that $X \equiv 0$ as well as $n \lambda=R$. Therefore, $M$ is an Einstein manifold and we finish the proof of the theorem.

4. Integral formulae for quasi-Einstein manifolds. In this section we shall show some integral formulae for a compact quasi-Einstein manifold $M^{n}$, which are generalisation of the formulae obtained for Ricci solitons in [1]. Those formulae enable us to derive some rigidity results for such a class of manifolds. 
THEOREM 3. Let $\left(M^{n}, g, \nabla f\right)$ be a Riemannian manifold satisfying $R i c_{\nabla f}^{m}=\lambda g$. Then we have

$$
\begin{aligned}
\frac{1}{2} \Delta_{f} R= & -\left|\nabla^{2} f-\frac{(\Delta f)}{n} g\right|^{2}-\frac{(\Delta f)^{2}}{n}+\lambda \Delta f+\frac{1}{2}\langle\nabla f, \nabla R\rangle+\frac{1}{2}\langle\nabla f, \nabla \Delta f\rangle \\
& +\frac{1}{m} \operatorname{div}\left(\nabla_{\nabla f} \nabla f-\Delta f \nabla f\right) .
\end{aligned}
$$

Proof. First of all we use Lemma 4 to obtain the following equation

$$
\begin{aligned}
\frac{1}{2} \Delta R-\frac{1}{2}\langle\nabla R, \nabla f\rangle= & -R i c(\nabla f, \nabla f)-\left|\nabla^{2} f-\frac{(\Delta f)}{n} g\right|^{2}-\frac{(\Delta f)^{2}}{n}+\lambda \Delta f+\frac{1}{2}\langle\nabla R, \nabla f\rangle \\
& +\frac{1}{m}|\nabla f|^{2} \Delta f+\frac{1}{m} \operatorname{div}\left(\nabla_{\nabla f} \nabla f-\nabla f \Delta f\right) .
\end{aligned}
$$

Now, using the definition of diffusion operator and substituting identity (1) of Corollary 2 in the preceding equation, we obtain

$$
\begin{aligned}
\frac{1}{2} \Delta_{f} R= & -\operatorname{Ric}(\nabla f, \nabla f)-\left|\nabla^{2} f-\frac{(\Delta f)}{n} g\right|^{2}-\frac{(\Delta f)^{2}}{n}+\lambda \Delta f \\
& +\frac{m-1}{m} \operatorname{Ric}(\nabla f, \nabla f)+\frac{1}{m}(R-(n-1) \lambda)|\nabla f|^{2}+\frac{1}{m}|\nabla f|^{2} \Delta f \\
& +\frac{1}{m} \operatorname{div}(\nabla \nabla f \nabla f-\nabla f \Delta f) .
\end{aligned}
$$

From here we deduce

$$
\begin{aligned}
\frac{1}{2} \Delta_{f} R= & -\left|\nabla^{2} f-\frac{(\Delta f)}{n} g\right|^{2}-\frac{(\Delta f)^{2}}{n}+\lambda \Delta f-\frac{1}{m} \operatorname{Ric}(\nabla f, \nabla f) \\
& +\frac{1}{m}(R+\Delta f-n \lambda)|\nabla f|^{2}+\frac{1}{m} \lambda|\nabla f|^{2}+\frac{1}{m} \operatorname{div}\left(\nabla_{\nabla f} \nabla f-\nabla f \Delta f\right) .
\end{aligned}
$$

Next, using $R+\Delta f-n \lambda=\frac{1}{m}|\nabla f|^{2}$, we infer

$$
\begin{aligned}
\frac{1}{2} \Delta_{f} R= & -\left|\nabla^{2} f-\frac{(\Delta f)}{n} g\right|^{2}-\frac{(\Delta f)^{2}}{n}+\lambda \Delta f \\
& +\frac{1}{m}\left\{-\operatorname{Ric}(\nabla f, \nabla f)+\frac{1}{m}|\nabla f|^{4}+\lambda|\nabla f|^{2}+\operatorname{div}\left(\nabla_{\nabla f} \nabla f-\nabla f \Delta f\right)\right\} .
\end{aligned}
$$

On the other hand, using equation (1.4) with $X=\nabla f$, we have

$$
-\operatorname{Ric}(\nabla f, \nabla f)+\frac{1}{m}|\nabla f|^{4}+\lambda|\nabla f|^{2}=\left\langle\nabla_{\nabla f} \nabla f, \nabla f\right\rangle=\frac{m}{2}(\langle\nabla f, \nabla R\rangle+\langle\nabla f, \nabla f\rangle),
$$

where for the last equality we have used equation (1.7). Substituting this in the above formula for $\Delta_{f} R$, we get the expression in the statement, which completes the proof of the theorem.

As a consequence of this theorem, we deduce the following integral formulae. 
Corollary 3. Let $\left(M^{n}, g, \nabla f\right)$ be a compact orientable Riemannian manifold satisfying $\operatorname{Ric}_{\nabla f}^{m}=\lambda g$. Then we have

(1) $\int_{M}\left|\nabla^{2} f-\frac{(\Delta f)}{n} g\right|^{2} \mathrm{~d} \mathrm{M}=\frac{3}{2} \int_{M}\langle\nabla f, \nabla R\rangle \mathrm{d} \mathrm{M}+\frac{n+2}{2 n} \int_{M}\langle\nabla f, \nabla \Delta f\rangle \mathrm{d}$.

(2) $\int_{M}\left|\nabla^{2} f-\frac{(\Delta f)}{n} g\right|^{2} \mathrm{dM}+\frac{n+2}{2 n} \int_{M}(\Delta f)^{2} \mathrm{dM}=\frac{3}{2} \int_{M}\langle\nabla f, \nabla R\rangle \mathrm{dM}$.

(3) $\int_{M} \operatorname{Ric}(\nabla f, \nabla f) \mathrm{d} \mathrm{M}+\frac{3}{2} \int_{M}\langle\nabla f, \nabla R\rangle \mathrm{dM}=\frac{3}{2} \int_{M}(\Delta f)^{2} \mathrm{~d} \mathrm{M}$.

(4) $M^{n}$ is an Einstein manifold, if $\int_{M}\langle\nabla R, \nabla f\rangle \mathrm{d} \mathbf{M} \leq 0$.

(5) Suppose that $f$ is not constant and there exists $\mu: M^{n} \rightarrow \mathbb{R}$ solution of the equation $\frac{n+2}{2 n} \Delta f+\frac{3}{2} R=\mu$, such that $\mu \perp \Delta f$, in the $L^{2}$ inner product. Then $M^{n}$ is conformally equivalent to a unit sphere $\mathbb{S}^{n}$, but not isometric.

Proof. Since $M^{n}$ is compact, we can use Theorem 3 and Stokes' formula to infer

$$
\begin{aligned}
\int_{M}\left|\nabla^{2} f-\frac{(\Delta f)}{n} g\right|^{2} \mathrm{~d} \mathrm{M}= & \int_{M}\left(\lambda-\frac{\Delta f}{n}\right) \Delta f \mathrm{~d} \mathrm{M}+\int_{M}\langle\nabla f, \nabla R\rangle \mathrm{dM} \\
& +\frac{1}{2} \int_{M}\langle\nabla f, \nabla(R+\Delta f)\rangle \mathrm{d} \mathrm{M} .
\end{aligned}
$$

Next, we use relation (1.6) to write $\int_{M}\left(\lambda-\frac{\Delta f}{n}\right) \Delta f \mathrm{dM}=\frac{1}{n} \int_{M}\left(R-\frac{1}{m}|\nabla f|^{2}\right) \Delta f \mathrm{dM}$. Then, Stokes' formula gives

$$
\frac{1}{n} \int_{M}\left(R-\frac{1}{m}|\nabla f|^{2}\right) \Delta f \mathrm{~d} \mathrm{M}=-\frac{1}{n} \int_{M}\langle\nabla f, \nabla R\rangle \mathrm{d} \mathrm{M}+\frac{1}{n m} \int_{M}\left\langle\nabla f, \nabla|\nabla f|^{2}\right\rangle \mathrm{d} \mathrm{M} .
$$

On the other hand, we notice that equation (1.6) yields $\nabla(R+\Delta f)=\frac{1}{m} \nabla\left(|\nabla f|^{2}\right)$. By using this datum on the previous equation, we have

$$
\int_{M}\left|\nabla^{2} f-\frac{(\Delta f)}{n} g\right|^{2} \mathrm{~d} \mathrm{M}=\frac{3}{2} \int_{M}\langle\nabla f, \nabla R\rangle \mathrm{d} \mathrm{M}+\frac{n+2}{2 n} \int_{M}\langle\nabla f, \nabla \Delta f\rangle \mathrm{d} \mathrm{M},
$$

which ends the first assertion.

Proceeding, since $\int_{M}\langle\nabla f, \nabla \Delta f\rangle \mathrm{dM}=-\int_{M}(\Delta f)^{2} \mathrm{dM}$, we obtain from equation (4.3) that

$$
\int_{M}\left|\nabla^{2} f-\frac{(\Delta f)}{n} g\right|^{2} \mathrm{~d} \mathrm{M}=\frac{3}{2} \int_{M}\langle\nabla f, \nabla R\rangle \mathrm{d} \mathrm{M}-\frac{n+2}{2 n} \int_{M}(\Delta f)^{2} \mathrm{dM},
$$

which gives the second item.

Next, we integrate Bochner's formula to get

$$
\int_{M} \operatorname{Ric}(\nabla f, \nabla f) \mathrm{d} \mathrm{M}+\int_{M}\left|\nabla^{2} f\right|^{2} \mathrm{dM}+\int_{M}\langle\nabla f, \nabla \Delta f\rangle \mathrm{dM}=0 .
$$

Since $\int_{M}\left|\nabla^{2} f-\frac{(\Delta f)}{n} g\right|^{2} \mathrm{dM}=\int_{M}\left|\nabla^{2} f\right|^{2} \mathrm{dM}-\frac{1}{n} \int_{M}(\Delta f)^{2} \mathrm{dM}$, we can use once more Stokes' formula to arrive at

$$
\int_{M} \operatorname{Ric}(\nabla f, \nabla f) \mathrm{d} \mathrm{M}+\int_{M}\left|\nabla^{2} f-\frac{(\Delta f)}{n} g\right|^{2} \mathrm{~d} \mathrm{M}=\frac{n-1}{n} \int_{M}(\Delta f)^{2} \mathrm{~d} \mathrm{M} .
$$


Now, comparing equation (4.6) with the second item we arrive at

$$
\int_{M}\left\{\operatorname{Ric}(\nabla f, \nabla f)+\frac{3}{2}\langle\nabla f, \nabla R\rangle\right\} \mathrm{dM}=\frac{3}{2} \int_{M}(\Delta f)^{2} \mathrm{dM},
$$

as we want.

On the other hand, if $\int_{M}\langle\nabla R, \nabla f\rangle \mathrm{d} \mathrm{M} \leq 0$, in particular this occurs if $R$ is constant, we deduce, from the second item, that

$$
\int_{M}\langle\nabla R, \nabla f\rangle \mathrm{d} \mathbf{M}=0
$$

and

$$
\int_{M}\left|\nabla^{2} f-\frac{(\Delta f)}{n} g\right|^{2} \mathrm{dM}+\frac{n+2}{2 n} \int_{M}(\Delta f)^{2} \mathrm{dM}=0 .
$$

This implies that $\nabla^{2} f=\frac{1}{n}(\Delta f) g$ and $\Delta f=0$. Hence, we can apply Hopf's theorem to deduce that $f$ is constant, which implies that $M^{n}$ is an Einstein manifold.

Finally, we notice that $\int_{M}\left|\nabla^{2} f-\frac{(\Delta f)}{n} g\right|^{2} \mathrm{dM}=\int_{M}\left\langle\nabla f, \nabla\left(\frac{n+2}{2 n} \Delta f+\frac{3}{2} R\right)\right\rangle \mathrm{dM}$. So, if $\frac{n+2}{2 n} \Delta f+\frac{3}{2} R=\mu$, with $\int_{M} \mu \Delta f \mathrm{dM}=0$, we have $\nabla^{2} f=\frac{1}{n}(\Delta f) g$. Since $f$ is not constant, this allows us to apply Theorem 2 due to Ishara and Tashiro [8] to conclude that $M^{n}$ is conformally equivalent to a unit sphere $\mathbb{S}^{n}$. Moreover, if we have an isometry between $M^{n}$ and $\mathbb{S}^{n}$, then its scalar curvature $R$ would be constant. From assertion (2), we conclude that $\int_{M}\left|\nabla^{2} f-\frac{(\Delta f)}{n} g\right|^{2} \mathrm{dM}+\frac{n+2}{2 n} \int_{M}(\Delta f)^{2} \mathrm{dM}=0$. Then, the previous assertion yields that $f$ must be constant, which contradicts our assumption on $f$. Hence, we complete the proof of the corollary.

As a consequence of this corollary, we derive the next result.

COROLlaRY 4. Let $\left(M^{n}, g, \nabla f\right)$ be an orientable compact Riemannian manifold satisfying $\operatorname{Ric}_{\nabla f}^{m}=\lambda g$. Then $\nabla f$ can not be a non-trivial conformal vector field.

Proof. Let us suppose that $\nabla f$ is a non-trivial conformal vector field, i.e. $\mathcal{L}_{\nabla f} g=$ $2 \rho g$ with $\rho$ not constant. Therefore, we can apply Theorem II.9 from [2] to deduce that

$$
\int_{M} \mathcal{L}_{\nabla f} R \mathrm{dM}=\int_{M}\langle\nabla f, \nabla R\rangle \mathrm{d} \mathrm{M}=0
$$

Then, the previous corollary enables us to finish the proof.

REMARK 2. We point out that $\int_{M}\langle\nabla f, \nabla R\rangle \mathrm{dM}=0$ in dimension two for $m$ finite is always valid. In fact, since $\nabla\left(e^{-\frac{f}{m}}\right)$ is a conformal field and the Dirichlet integral is a conformal invariant, the claim follows from Theorem II.9 from [2]. Therefore, if $\left(M^{2}, g, \nabla f\right)$ is a compact quasi-Einstein manifold, then it is trivial by Corollary 3 , see also [5] and [9].

ACKNOWLEDGEMENT. The authors would like to thank the referee for comments and valuable suggestions. 


\section{REFERENCES}

1. C. Aquino, A. Barros and E. Ribeiro, Jr, Some applications of the Hodge-de Rham decomposition to Ricci solitons, Results Math. 60 (2011), 245-254. Doi:10.1007/s0002501100166-1.

2. J. P. Bourguignon and J. P. Ezin, Scalar curvature functions in a conformal class of metrics and conformal transformations, Trans. Am. Math. Soc. 301 (1987), 723-736.

3. F. Camargo, A. Caminha and P. Souza, Complete foliations of space forms by hypersurfaces, Bull. Braz. Math. Soc. 41 (2010), 339-353. $227-284$.

4. J. Case, On the nonexistence of quasi-Einstein metrics, Pacific J. Math. 248 (2010), 93-100.

5. J. Case, Y. Shu and G. Wei, Rigity of quasi-Einstein metrics, Diff. Geo. Appl. 29 (2010),

6. M. Eminenti, G. La Nave and C. Mantegazza, Ricci solitons: The equation point of view, Manuscripta Math. 127 (2008), 345-367.

7. R. S. Hamilton, The formation of singularities in the Ricci flow, Surv. Diff. Geom. 2 (1993), 7-136 (International Press, Cambridge, MA).

8. S. Ishihara and Y. Tashiro, On Riemannian manifolds admitting a concircular transformation, Math. J. Okayama Univ. 9 (1959), 19-47.

9. D. S. Kim and Y. H. Kim, Compact Einstein warped product spaces with nonpositive scalar curvature, Proc. Am. Math. Soc. 131 (2003), 2573-2576. 329-345.

10. P. Petersen and W. Wylie, Rigidity of gradient Ricci solitons, Pacific J. Math. 241 (2009),

11. S. T. Yau, Some function-theoretic properties of complete Riemannian manifolds and their applications to geometry, Indiana Univ. Math. J. 25 (1976), 659-670. 